\title{
Current state of the art and future of myectomy
}

\author{
Magdi H. Yacoub ${ }^{1,2}$, Ahmed Afifi', Hesham Saad ${ }^{2}$, Heba Aguib $^{1,2}$, Ahmed ElGuindy $^{1,2}$ \\ ${ }^{1}$ National Heart and Lung Institute, Imperial College London, London, UK; ${ }^{2}$ Aswan Heart Centre, Aswan Governorate, Egypt \\ Correspondence to: Magdi H. Yacoub, FRS. Heart Science Centre, National Heart and Lung Institute, Imperial College London, London, UK. Email: \\ m.yacoub@imperial.ac.uk.
}

\begin{abstract}
Surgical relief of left ventricular outflow tract obstruction (LVOTO) in hypertrophic cardiomyopathy (HCM) requires more than septal myectomy. The procedure is currently the gold standard for all symptomatic HCM patients except those with comorbidities. The operation requires an individualized approach to restore the sophisticated functions of the left ventricular outflow tract (LVOT) without injury to the surrounding vital structures. The procedure should be tailored to deal with all the varied components of the obstruction including fibrous trigones, accessory tissues, and papillary muscle fusion. Preoperative multimodality imaging and numerical modeling combined with intraoperative transesophageal echocardiography (TEE) are essential to define existing anomalies as well as assess the adequacy of the repair. The mitral valve can be conserved in virtually all patients. The operation can be conducted with very low mortality and morbidity with predictable good outcomes both in the short and long term. Nevertheless, surgical relief of LVOTO is still grossly underused.
\end{abstract}

Keywords: Myectomy; hypertrophic cardiomyopathy (HCM); HOCM

Submitted Apr 05, 2017. Accepted for publication Jun 02, 2017.

doi: $10.21037 /$ acs.2017.06.04

View this article at: http://dx.doi.org/10.21037/acs.2017.06.04

\section{Introduction}

Hypertrophic cardiomyopathy (HCM) is extremely heterogeneous with regards to both genetic origins and phenotypic expression $(1,2)$. One of the major features of the disease is the development of left ventricular outflow obstruction (3) which, if unrelieved, influences prognosis, in terms of both quality of life and survival. The anatomic and functional changes contributing to left ventricular outflow tract obstruction (LVOTO) in HCM varies from one patient to another and necessitates an individualized restorative approach. This requires a thorough understanding of the sophisticated structure/ function relationship of the aortic root in health and in HCM, followed by defining the specific abnormalities that need to be dealt with in each patient $(4,5)$.

We here describe the functional and surgical pathology of the left ventricular outflow tract (LVOT), followed by a systematic account of the currently performed restorative operation and speculation about its future (Video 1).

\section{Functional and surgical pathology of the LVOT in HCM}

The left ventricular outflow and aortic root occupy a very strategic location in relation to the left ventricle, and the entire arterial system. They perform sophisticated vital functions, including the previously neglected reservoir function, which depend on its complex geometry and dynamism of the component parts (6). The LVOT shares the same orifice, in the flask-shaped left ventricular mass, with the mitral valve (Figures 1,2). This enables the $L V$ to deliver a bolus of blood rapidly, under high pressure, into the systemic circulation.

The aortic and mitral orifices are separated by the sub-aortic curtain, which moves during the cardiac cycle to allow the aortic orifice to enlarge during systole and the mitral valve during diastole (Figures 1,2). The hinge mechanism allowing this movement consists of two components of the fibrous network of the heart (7), termed the right and left fibrous trigones (Figures 1,2). The right 

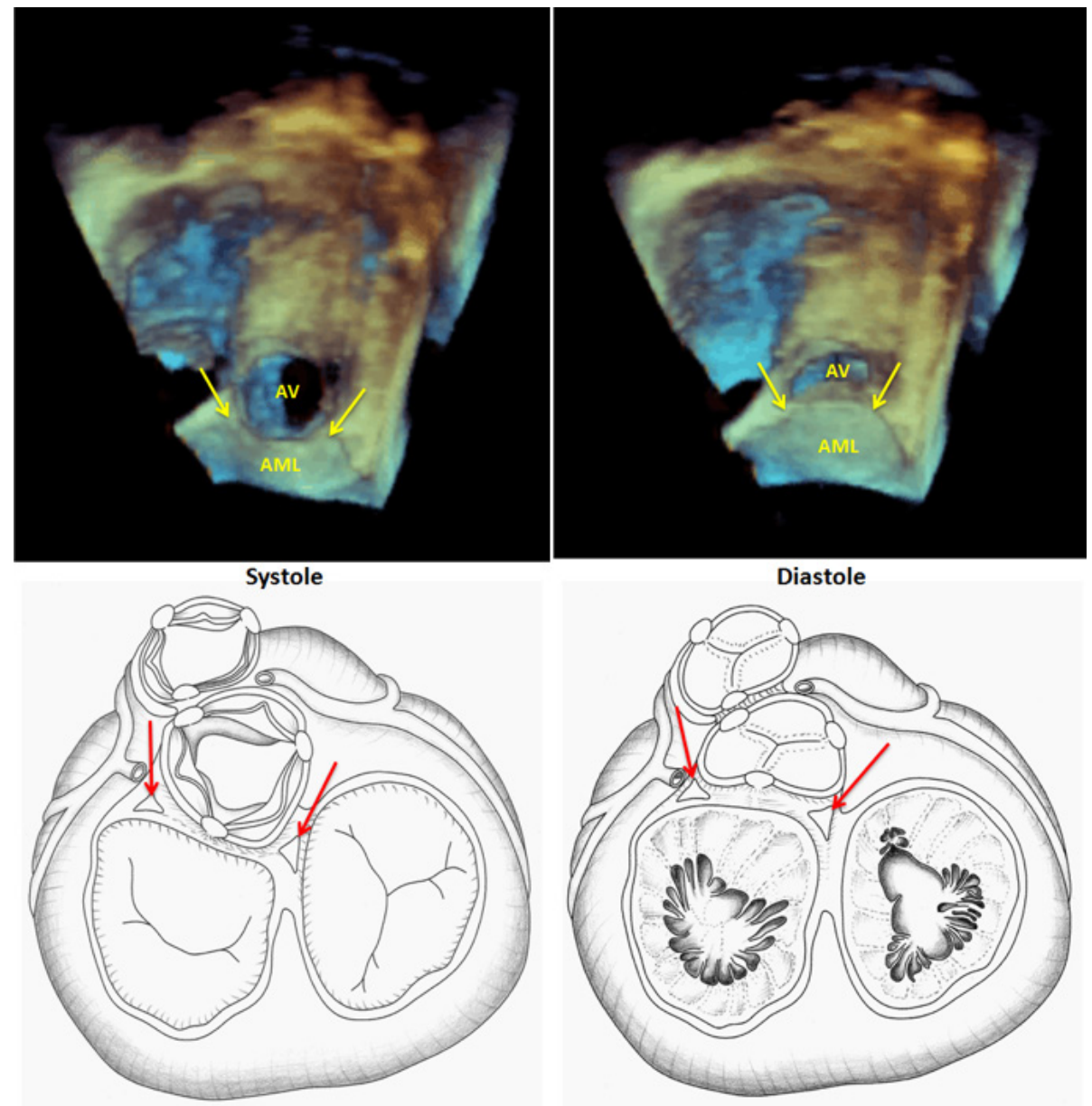

Figure 1 The LVOT and the mitral valve annulus share a common opening which allows, by aorto-mitral interdependence, for optimal flow through their orifices in systole and diastole respectively (5). LVOT, left ventricular outflow tract.

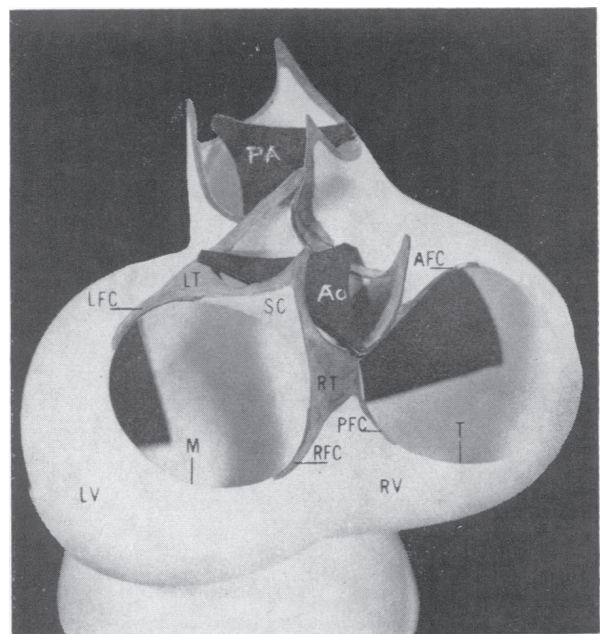

Figure 2 Relationship between the aortic and mitral valve annulus showing the fibrous trigones and subaortic curtain (7). fibrous trigone, or central fibrous body of the heart, is located at the junction of the sub-aortic membrane to the membranous inter-ventricular septum on the ventricular side and the posterolateral commissure of the mitral valve on the atrial side. The right fibrous trigone is also closely related to the septal leaflet of the tricuspid valve and the atrioventricular bundle of His. The left fibrous trigone is located at the attachment of the sub-aortic curtain to the muscular inter-ventricular septum at the postero-lateral angle of the LVOT, and the antero-lateral commissure of the mitral valve (Figures 2,3).

An understanding of the normal anatomy of the fibrous trigones, and importantly, the changes that occur in many patients with LVOTO due to HCM is fundamental to achieving optimal obstructive relief. Abnormalities of the left fibrous trigone are more common than those of the 


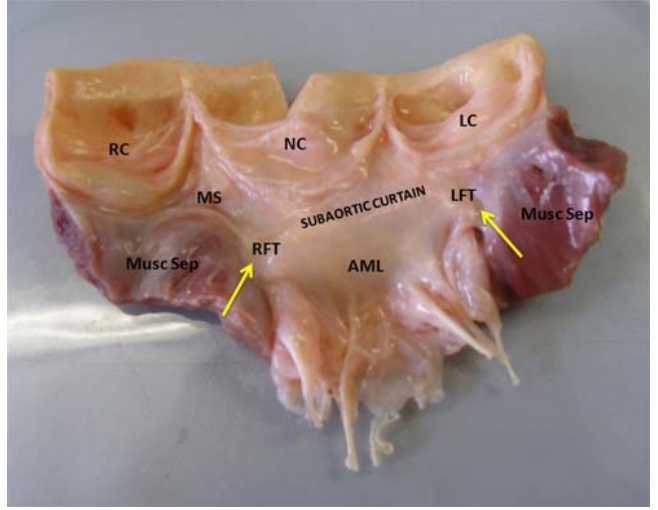

Figure 3 Surgically resected specimen of the LVOT lay open to show the relationship between its component parts (5). RC, right cusp; NC, non-coronary cusp; LC, left cusp; MS, membranous septum; Musc Sep, muscular septum; AML, anterior mitral leaflet; LVOT, left ventricular outflow tract.

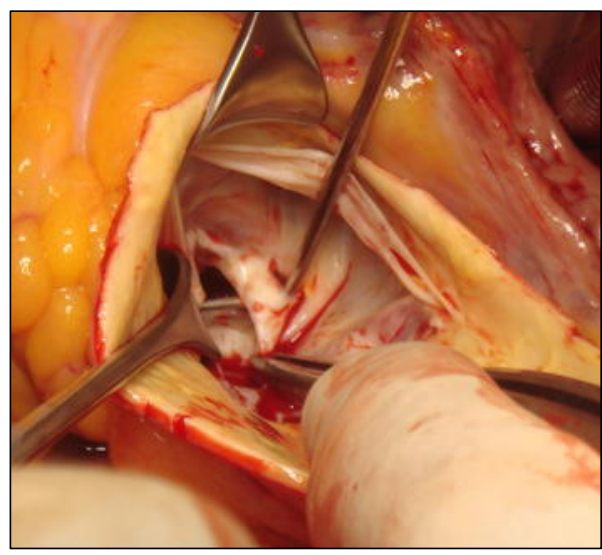

Figure 4 Release of the left fibrous trigone by blunt dissection, with peeling of shelf-like fibrous adhesions.

right. The commonest of these is the fusion between the bulging muscular septum and the fibrous trigone, resulting in a shelf-like fibrous membrane. This membrane can continue onto the muscular septum and/or the anterior leaflet of the mitral valve, in a manner similar to that encountered in fixed subaortic fibrous ring, but which does not form a complete ring. The shelf may extend into the ventricular cavity, tethering the lateral aspect of the mitral apparatus to the muscular inter-ventricular septum. The fusion of the left fibrous trigone prevents the subaortic curtain from moving backwards during systole and can therefore contribute significantly to LVOTO. The shelf- like fusion can be easily peeled off using a blunt hook (Figure 4). Similar changes to those observed at the left fibrous trigone can be seen, less commonly, at the right fibrous trigone. This results in a similar shelf-like structure fixing the subaortic curtain to the membranous inter ventricular septum (IVS). Great care should be taken during peeling of the shelf due to the proximity to the conducting tissue.

The muscular inter ventricular septum (MIVS) forms the anterior wall of the LVOT and extends from the left fibrous trigone to the membranous septum in the horizontal plane, and from the attachment of the aortic valve above to a level just below of the base of the papillary muscles. It is the bulge of the IVS into the LVOT that is responsible for the initial major component of the obstruction to the LVOT. The shape of this bulge can be defined by MRI, and can be described as oval or sigmoid (8) (Figure 5).

The MIVS is markedly thickened in HCM, measuring up to $4 \mathrm{~cm}$ in the parasternal long axis view of an echocardiogram. The thickness of the posterior wall is usually also increased, but to a less extent, constituting the classical "asymmetric septal hypertrophy". The endocardial surface of the MIVS, in the area of obstruction, is covered by a patch of glistening, white sub-endocardial fibrosis due to the repeated trauma produced by the anterior leaflet of the mitral valve (Figure 6).

The MIVR is the major target for the myectomy operation, where trans-aortic "extended resection" of the obstructive bulge is performed over a region extending from $2 \mathrm{~mm}$ to the left of the membranous septum, to the left fibrous trigone in the horizontal plane, and from just below the aortic valve to a level below the attachment of the papillary muscles (Figure 6). The thickness of the resected muscle varies according to the measurements of the MIVS, determined intraoperatively by transesophageal echocardiography (TEE), and includes the obstructive muscle bands (Figure 7).

\section{The mitral valve apparatus in HCM}

Another major player in the obstructive process is the mitral valve apparatus, which forms the posterior boundary of the LVOT. Although several primary and secondary structural and functional abnormalities are present in HOCM, only a few require direct operations on the mitral valve and are completely reversed by myectomy alone. A typical example of that is systolic anterior motion of the mitral valve SAM (Figure $7 A$ ), which is very frequently present, contributes to 

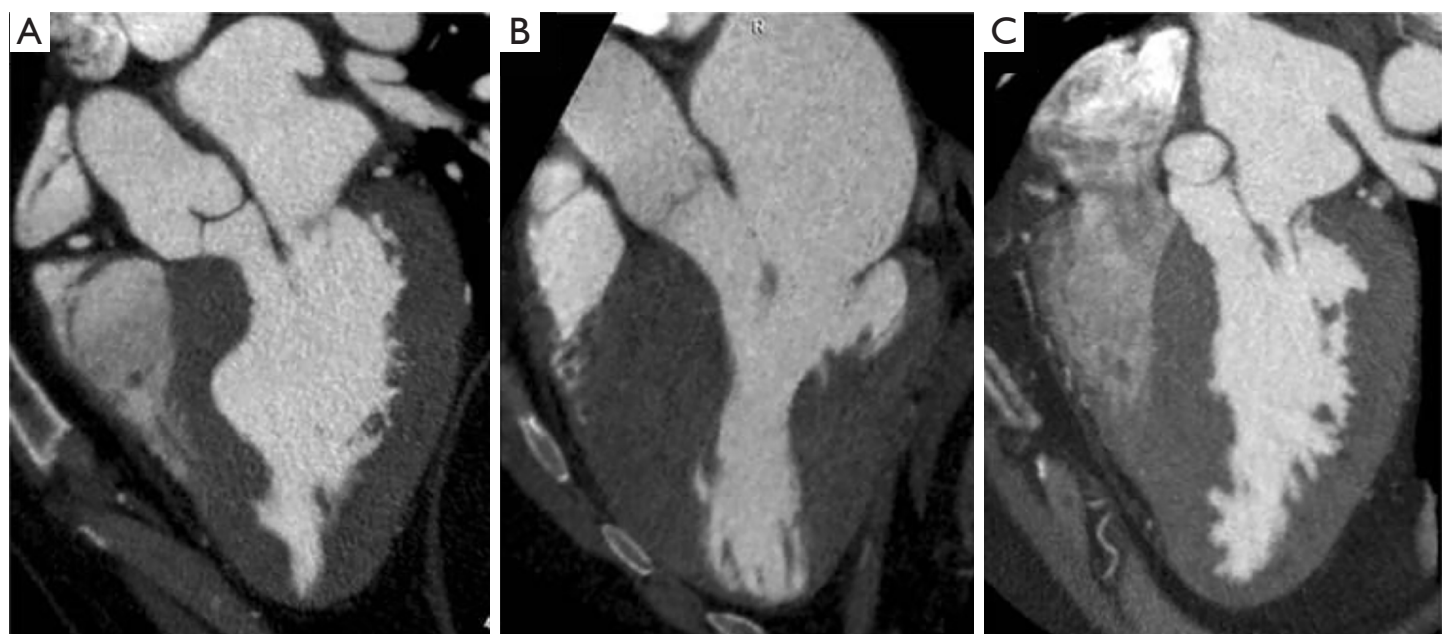

Figure 5 The different morphologies of the septal hypertrophy in HOCM as seen by computed tomography (5). (A) Sigmoid septum; (B) reverse curve septum; (C) neutral septum).

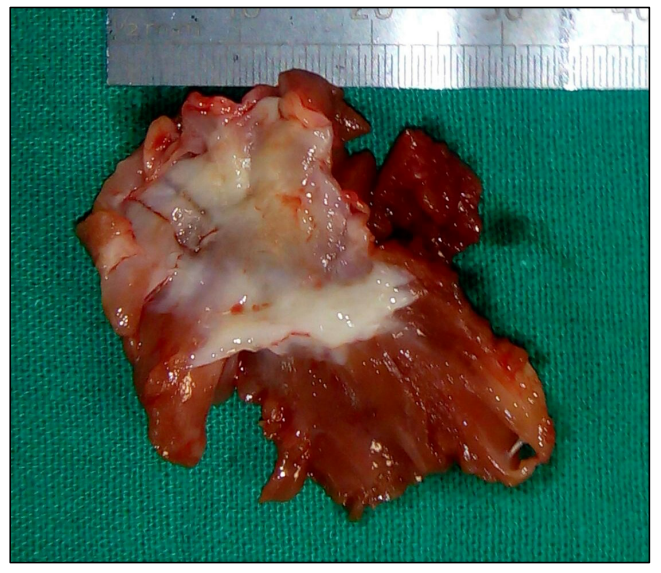

Figure 6 Surgical excision of the septal bulge in the muscular IVS seen from its endocardial surface. At the area of obstruction, is covered by a patch of glistening, white sub-endocardial fibrosis from repeated trauma produced by the anterior leaflet of the mitral valve and the high velocity jet of blood. IVS, inter ventricular septum.

both obstruction and mitral regurgitation, and disappears completely following optimally performed extended myectomy (Figure $7 B$ ). The long anterior leaflet, which is thought to be one of the primary abnormalities does not require direct operations on the mitral valve.

The abnormalities that require direct operation on the mitral valve are less common and include:

(I) Abnormal chordae attaching the mitral valve or the papillary muscles to the septum or free ventricular wall.

(II) Fusion of the anterolateral papillary muscle to the free ventricular wall.

(III) Flail segments of one or both mitral leaflets. This is extremely rare and almost certainly represents the coexistence of two different diseases.

(IV) Massive hypertrophy of one or both papillary muscles, contributing to the formation of a "sphincter" at the mid ventricular level. In these cases, it is preferable to deal with other components of the "sphincter" rather than thinning the papillary muscles.

\section{The ascending aorta}

Several studies have documented increased fibrosis and deranged biomechanical properties in patients with HCM. The cause, extent and significance of these changes need to be investigated further (9).

\section{The coronary circulation}

Coronary microvascular compromise (CMC) in HCM is a progressive condition which has been shown to be an important prognostic indicator (10). One of the important factors contributing to CMC in HCM is related to "contraction-coronary coupling" as shown by wave intensity analysis (11) (Figure 8). 

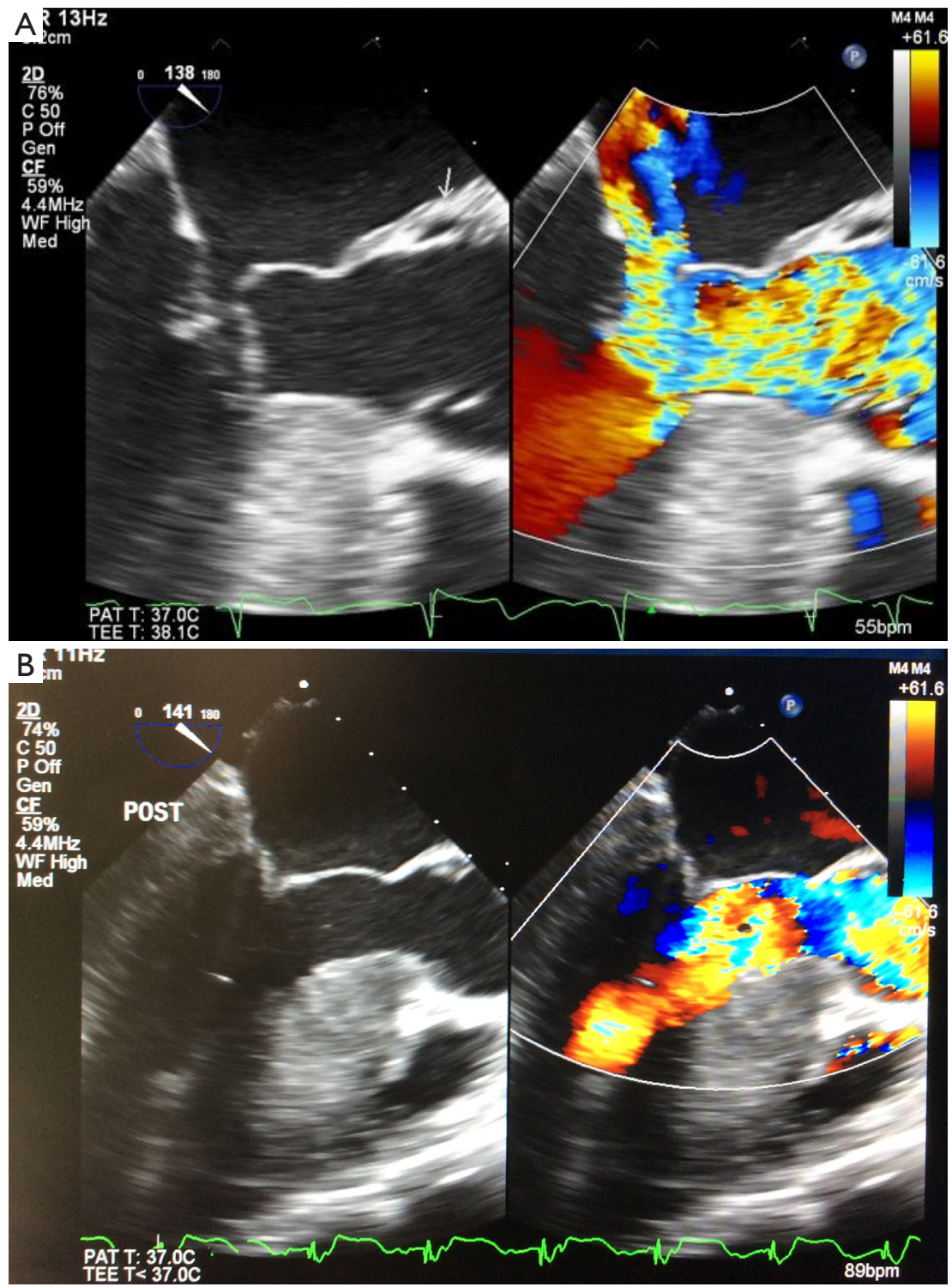

Figure 7 Pre- and post-myectomy TEE images. (A) Trans-esophageal echocardiography (mid-esophageal long axis view) showing the septal bulge of HOCM covered by a glistening endocardium denoting fibrosis, severe systolic anterior motion of both leaflets of the mitral valve and an apical to basal band; (B) post-operative trans-esophageal echocardiography (mid-esophageal long axis view) of the same patient, showing how resection of the septal bulge and mobilization of the fibrous trigones eliminates systolic anterior motion of the mitral valve. TEE, transesophageal echocardiography.

This type of CMC is markedly aggravated by LVOTO and is expected to improve immediately after relief of LVOTO in a manner similar to that observed after relief of aortic stenosis (12). Further improvement in the coronary microcirculation after relief of LVOTO can occur due to reverse myocardial remodeling, which occurs after myectomy (13).
In addition, there is evidence that unrelieved LVOTO can result in adverse remodelling and myocardial fibrosis, which are associated with coronary dysfunction in HCM (14), as well as clinical deterioration resulting in "end stage HCM" $(15,16)$. Asymptomatic patients with large gradients across LVOT could be at risk of developing irreversible ischemic myocardial damage. The utility 

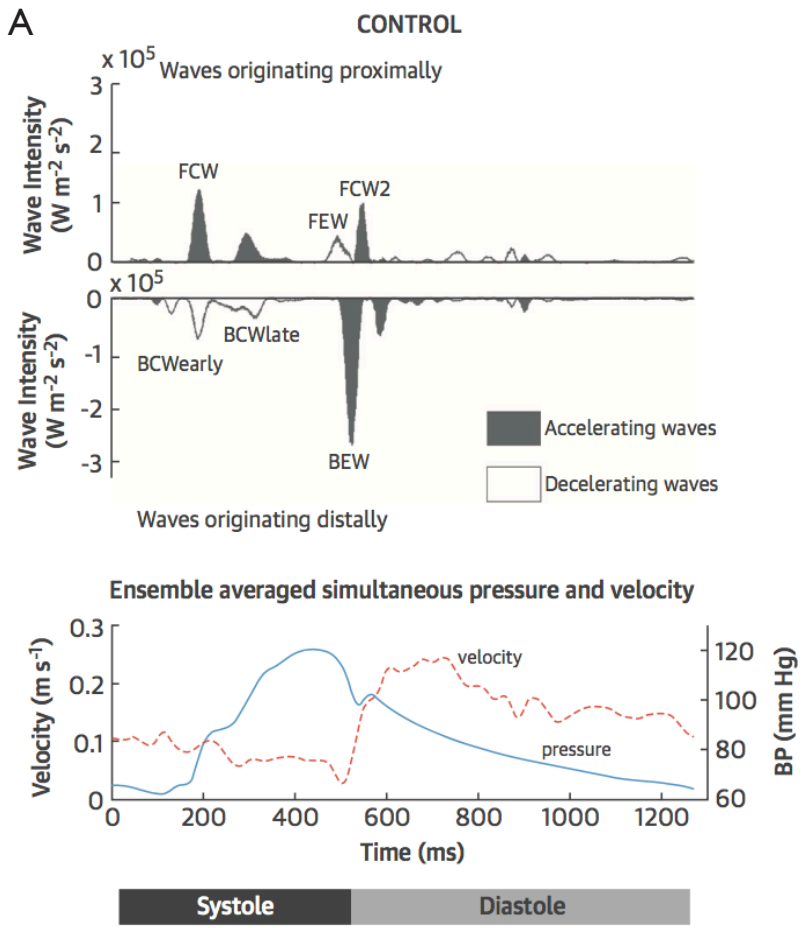
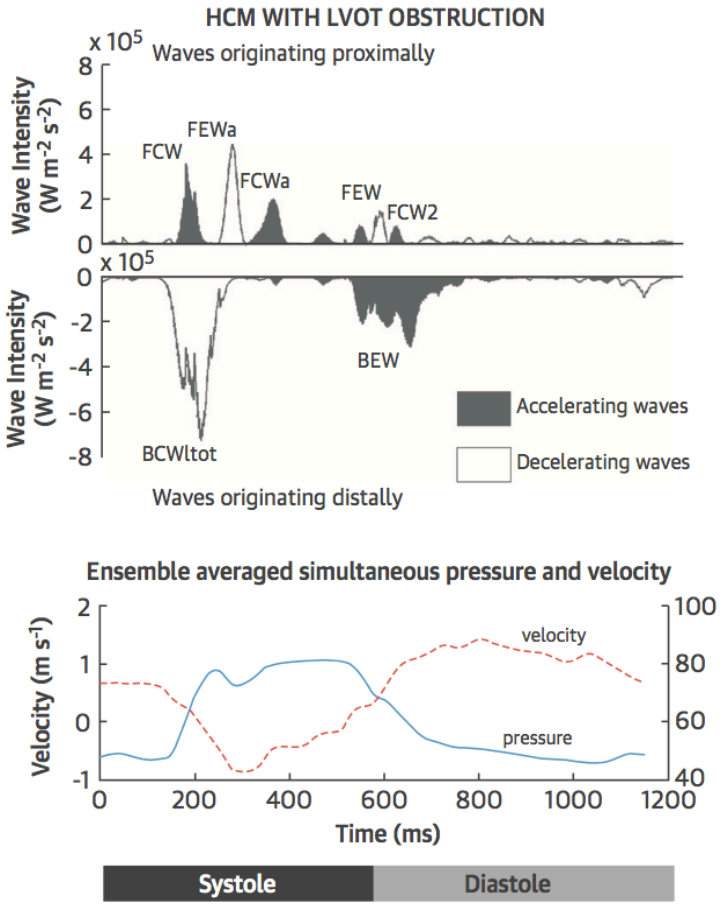

B
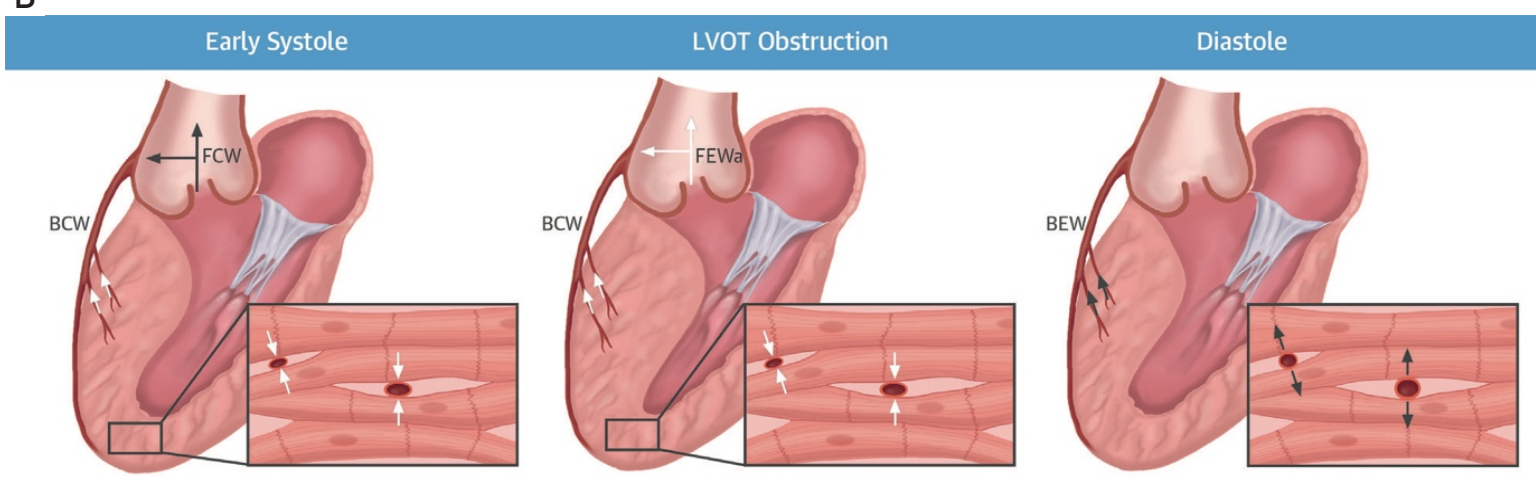

Figure 8 Potential influence of relief of left ventricular outflow tract obstruction on coronary microcirculation using wave intensity analysis. (A) Effect of positive and negative waves; (B) cartoon explaining the mechanisms involved.

of prophylactic myectomy in these patients needs to be investigated in a prospective randomized trial.

\section{Myocardial bridges and tunnels}

Another potentially important cause of myocardial ischemia in HCM is the not uncommon coexistence of compression of one or more major epicardial coronary arteries by myocardial bridges or tunnels (17). These can be easily detected by preoperative selective coronary angiography and CT angiography. The two techniques are complementary, as the first shows "milking" of a segment of the artery (Videos 2,3) while the latter shows the thickness of the bridge or tunnel. The relevance of such a finding can be defined further by measuring diastolic FFR (18), which can help in deciding whether such a segment needs de-tunnelling at the time of myectomy (Figure 9).

\section{A tailored surgical approach}

\section{Pre-operative assessment and planning}

The current indication for myectomy is significant LVOT 

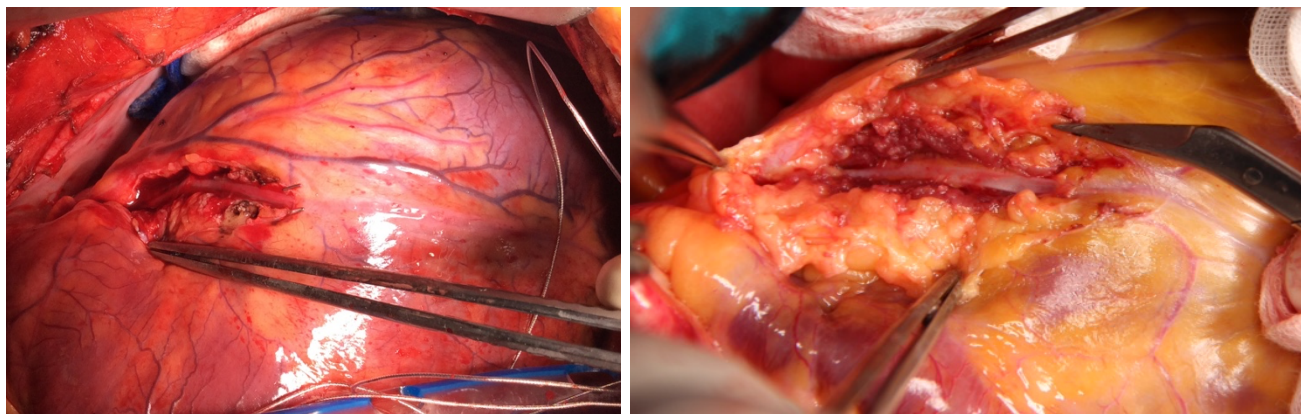

Figure 9 De-tunnelling of the left anterior descending artery.

obstruction (resting or provoked gradient $>50 \mathrm{mmHg}$ and NYHA class III or IV symptoms and/or recurrent exerciseinduced syncope despite maximally tolerated medical treatment) (19).

Planning the particulars of the personalized operation requires a series of tests (Table 1), imaging procedures and possibly three-dimensional modeling of both the cavity and the LV muscle mass (Figure 10).

\section{Surgical technique and post-operative management}

Perioperative trans-esophageal echocardiography is used to determine the thickness of the septum and the distance of the maximum thickness of septal bulge from the aortic annulus. The degree of systolic anterior motion, and the degree of mitral regurgitation is quantified (Figure 7). The site and severity of the existing obstruction are measured before and after myectomy. In addition, the existence of anomalous chordae or muscle bands is defined.

Antegrade cold blood cardioplegia is used for myocardial protection. The LVOT is widely exposed through a hockey stick incision in the ascending aorta extending into the noncoronary sinus (Figure 4).

Following detailed inspection, repair is started by release of the fibrous trigones. The anterior mitral leaflet is retracted to expose its junction to the interventricular septum on either side, thus exposing trigonal fusion. Blunt dissection using a nerve hook releases the shelflike obstruction and its extension onto the septum or anterior mitral leaflet. Additional sharp dissection may be required, taking care not to injure the conducting tissue or the mitral valve apparatus. The septal myectomy incision is performed (using an 11 blade) starting $0.5-1 \mathrm{~cm}$ from below the aortic annulus from the region of the left fibrous trigone, extending clockwise to a point $3 \mathrm{~mm}$ to the left of the membranous septum to avoid the conducting tissue. The top of the mobilized muscle to be excised is "grabbed" using a biopsy forceps, and the incision in the septum is extended into the ventricular cavity, developing a plane parallel to the endocardial surface to a level below the insertion of the papillary muscles. The thickness of the excised muscle is guided by the perioperative echo cardiographic measurements. The width of the myectomy is determined by the vertical incisions, which should diverge laterally into the depth of the ventricular cavity to include all the subendocardial fibrous thickening and the three obstructive muscle bands (Figure 6).

Any existing abnormal mitral chordate are divided and papillary muscle that are fused to the free ventricular wall are mobilized. In patients with myocardial bridges or tunnels causing compromise of the coronary microcirculation, surgical "de-tunnellings" is performed (Figure 9).

Following discontinuation of cardiopulmonary bypass and appropriate ventricular filling, any residual obstruction is measured, its cause determined, and surgical correction is considered. The use of inotropic drugs is avoided as they can create obstruction.

\section{Surgical results}

Despite the massive need for surgical myectomy worldwide and robust evidence for the effectiveness and superiority of the operation, application of this technique is inadequate and very sporadic (21). This could be due to the lack of familiarity and experience with the operation and the reported very high mortality of the operation reported by centers performing small numbers per year (22). The early results of the operation performed in experienced centers are excellent references. This is confirmed by our 
Table 1 Key components of preoperative assessment of potential myectomy candidates

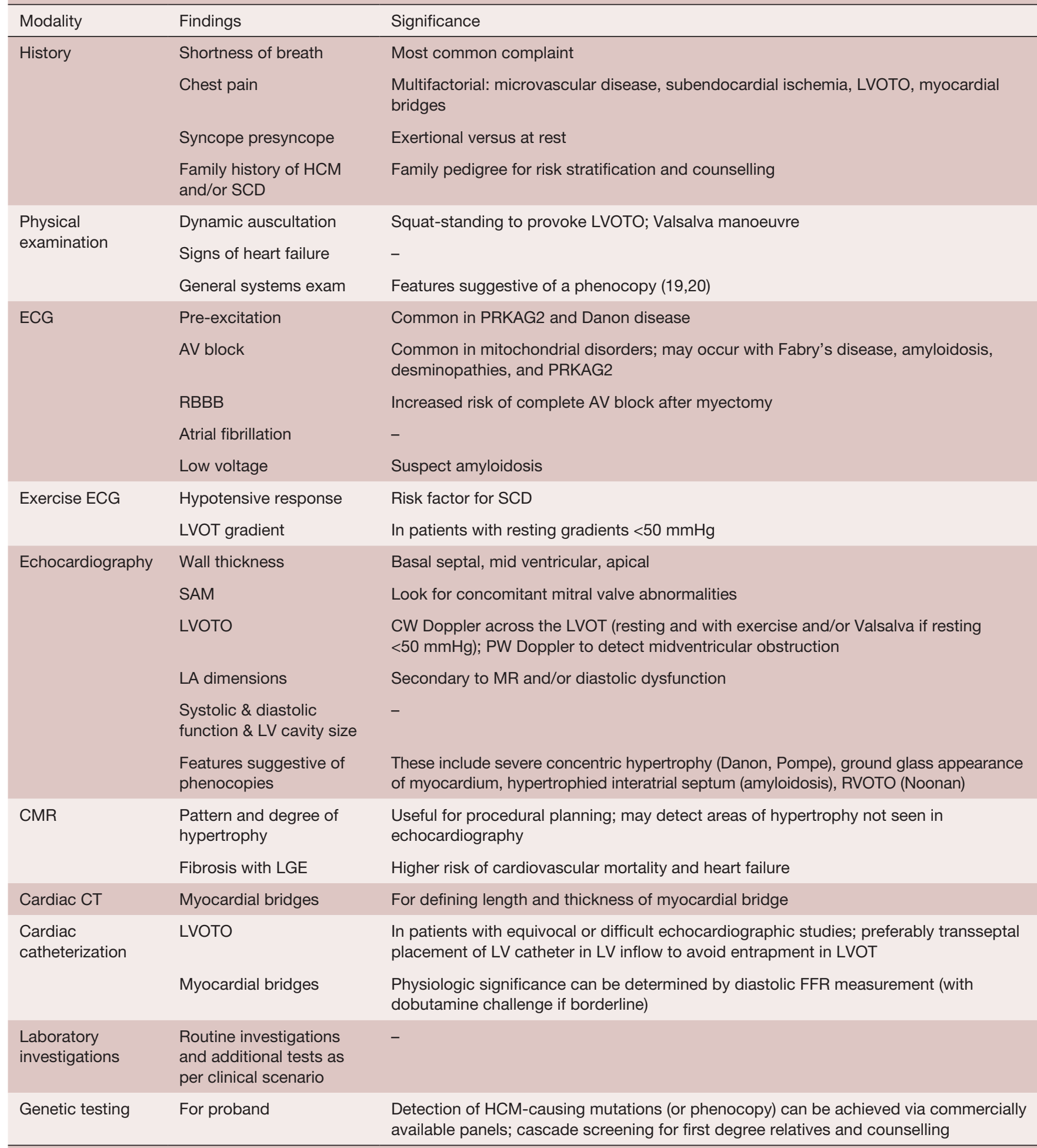

LVOTO, left ventricular outflow tract obstruction; HCM, hypertrophic cardiomyopathy; SCD, sudden cardiac death; RBBB, right bundle branch block; AV, atrio ventricular; SAM, systolic anterior motion; CW, continuous wave; PW, pulsed wave; MR, mitral regurgitation; LA, left atrium; LV, left ventricle; RVOTO, right ventricular outflow tract obstruction; CMR, cardiac magnetic resonance; LGE, late gadolinium enhancement; CT, computed tomography; FFR, fractional flow reserve. 

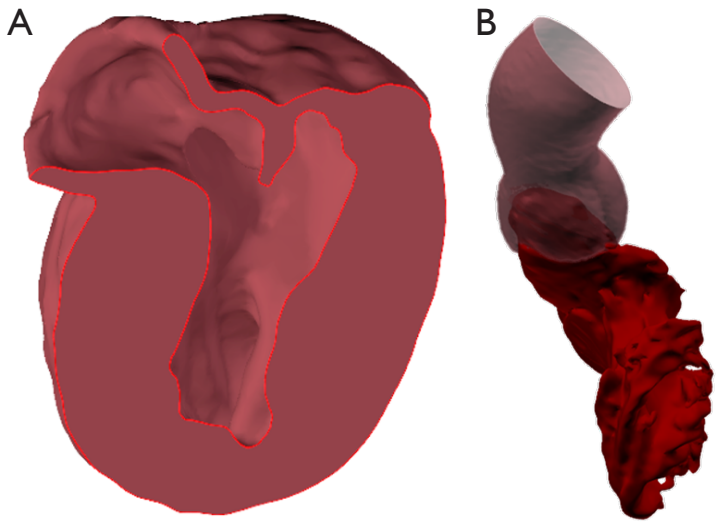

Figure 10 Three-dimensional modelling of the left ventricular muscle mass (A) and cavity (B).

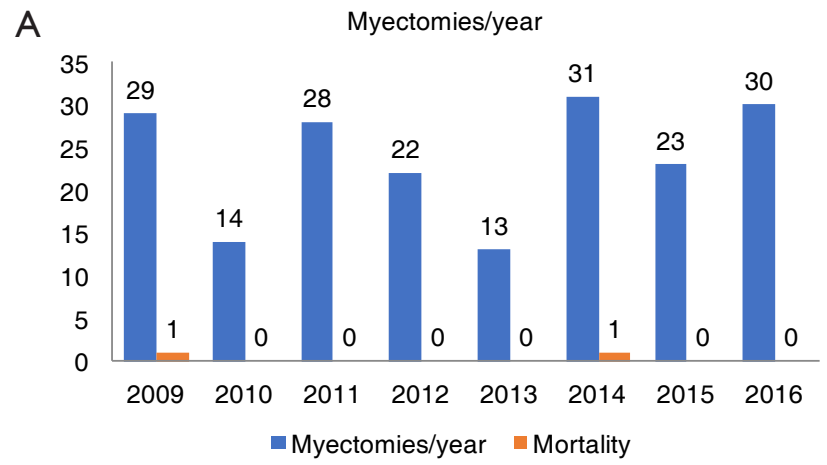

experience at the Aswan Heart Centre (Figure 11).

Similarly, long term studies have shown that the operation improves the survival of patients with obstruction, to be similar to those of the normal population (23) (Figure 12).

\section{Future of myectomy}

The use of currently available and emerging imaging modalities, such as diffusion tensor MRI (DTI) (24), could be applied to define the deranged pattern of myocardial trabeculae and possibly fiber orientation, in individual patients, to further enhance the precision of the operation. In addition, 3D modelling of the ventricle coupled with application of virtual operations with more detailed analysis

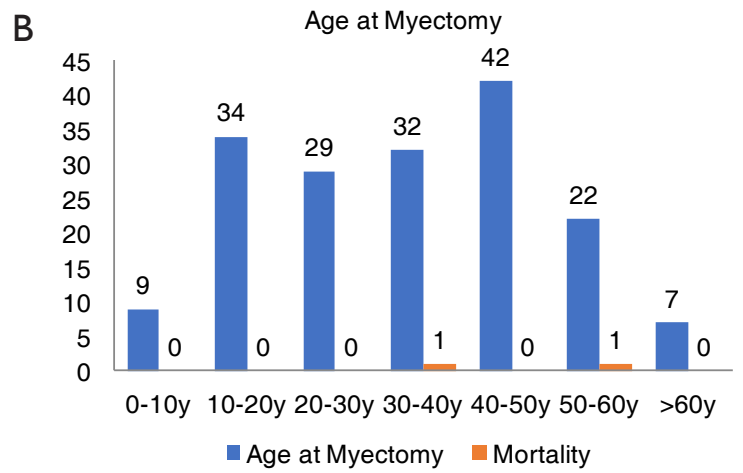

Figure 11 Aswan Heart Centre experience. (A) Annual number of myectomies and early mortality; (B) age of patients undergoing myectomy collectively with their age-related mortality.
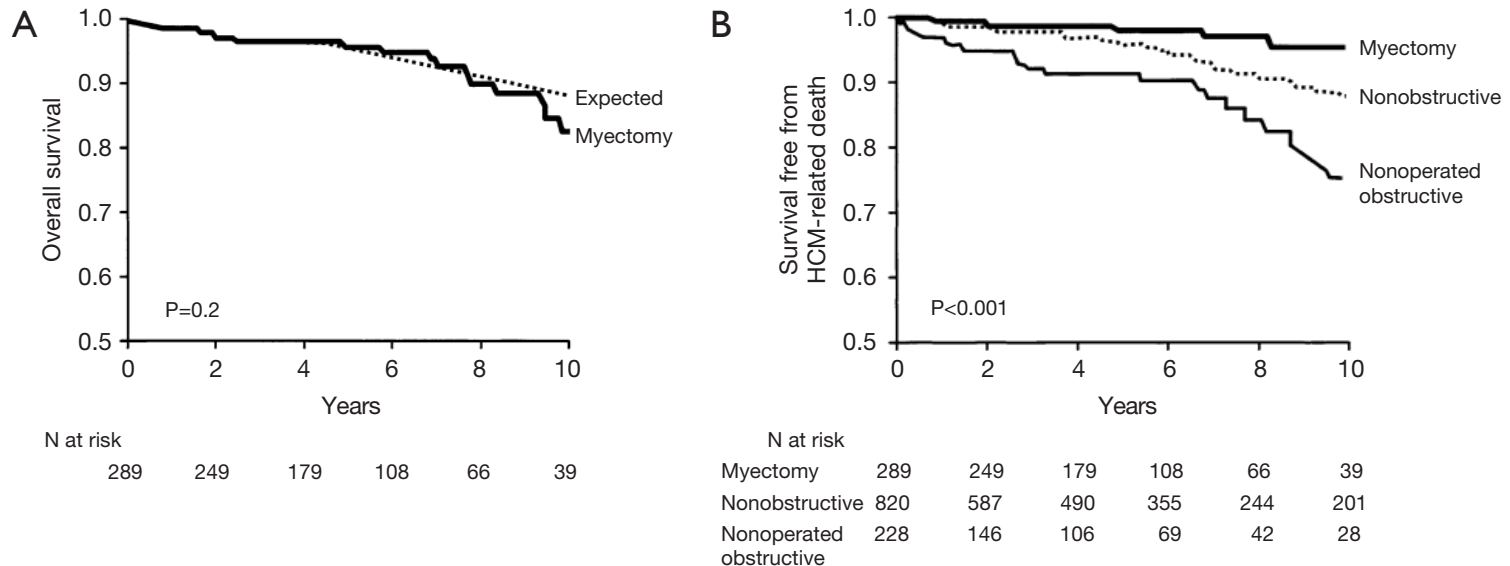

Figure 12 Survival benefit for myectomy. (A) Survival free from all-cause mortality after surgical myectomy for obstructive hypertrophic cardiomyopathy compared with the age- and gender-matched general U.S. white population; (B) survival free from HCM-related death among patients in three HCM subgroups: surgical myectomy, non-operated with obstruction, and nonobstructive (23). HCM, hypertrophic cardiomyopathy. 
of the possible effects on the hemodynamics (25) and possibly the kinetics of the myocardium may be of value in further refinement of the operation (26). The development of instruments to enhance visibility and precision inside the ventricular cavity could be of assistance during operation.

In the short and medium term, there is a pressing need for increasing and broadening the application of this operation to optimize the outcome of patients with LVOTO.

\section{Acknowledgements}

The authors thank Kholoud Salah for the video editing. Funding: This work is part of an ongoing program funded by the Egyptian Science and Technology Development (grant number 5309).

\section{Footnote}

Conflicts of Interest: The authors have no conflicts of interest to declare.

\section{References}

1. Braunwald E. Hypertrophic cardiomyopathy: The first century 1869-1969. Glob Cardiol Sci Pract 2012;2012:5.

2. Yacoub MH. Decade in review--cardiomyopathies: Cardiomyopathy on the move. Nat Rev Cardiol 2014;11:628-9.

3. Maron MS, Olivotto I, Zenovich AG, et al. Hypertrophic cardiomyopathy is predominantly a disease of left ventricular outflow tract obstruction. Circulation 2006;114:2232-9.

4. Yacoub MH, Kilner PJ, Birks EJ, et al. The aortic outflow and root: a tale of dynamism and crosstalk. Ann Thorac Surg 1999;68:S37-43.

5. Yacoub MH, El-Hamamsy I, Said K, et al. The left ventricular outflow in hypertrophic cardiomyopathy: from structure to function. J Cardiovasc Transl Res 2009;2:510-7.

6. Davies JE, Hadjiloizou N, Leibovich D, et al. Importance of the aortic reservoir in determining the shape of the arterial pressure waveform - The forgotten lessons of Frank. Artery Research 2007;1:40-5.

7. Zimmerman J. The functional and surgical anatomy of the heart. Ann R Coll Surg Engl 1966;39:348-66.

8. Olivotto I, Girolami F, Nistri S, et al. The many faces of hypertrophic cardiomyopathy: from developmental biology to clinical practice. J Cardiovasc Transl Res
2009;2:349-67.

9. Boonyasirinant T, Rajiah P, Setser RM, et al. Aortic stiffness is increased in hypertrophic cardiomyopathy with myocardial fibrosis: novel insights in vascular function from magnetic resonance imaging. J Am Coll Cardiol 2009; 54:255-62.

10. Maron MS, Olivotto I, Maron BJ, et al. The case for myocardial ischemia in hypertrophic cardiomyopathy. J Am Coll Cardiol 2009;54:866-75.

11. Raphael CE, Cooper R, Parker KH, et al. Mechanisms of Myocardial Ischemia in Hypertrophic Cardiomyopathy: Insights From Wave Intensity Analysis and Magnetic Resonance. J Am Coll Cardiol 2016;68:1651-60.

12. Rolandi MC, Wiegerinck EM, Casadonte L, et al. Transcatheter Replacement of Stenotic Aortic Valve Normalizes Cardiac-Coronary Interaction by Restoration of Systolic Coronary Flow Dynamics as Assessed by Wave Intensity Analysis. Circ Cardiovasc Interv 2016;9:e002356.

13. El-Hamamsy I, Lekadir K, Olivotto I, et al. Pattern and degree of left ventricular remodeling following a tailored surgical approach for hypertrophic obstructive cardiomyopathy. Glob Cardiol Sci Pract 2012;2012:9.

14. Petersen SE, Jerosch-Herold M, Hudsmith LE, et al. Evidence for microvascular dysfunction in hypertrophic cardiomyopathy: new insights from multiparametric magnetic resonance imaging. Circulation 2007;115:2418-25.

15. Olivotto I, Cecchi F, Poggesi C, et al. Patterns of disease progression in hypertrophic cardiomyopathy: an individualized approach to clinical staging. Circ Heart Fail 2012;5:535-46.

16. Yacoub MH, Olivotto I, Cecchi F. 'End-stage' hypertrophic cardiomyopathy: from mystery to model. Nat Clin Pract Cardiovasc Med 2007;4:232-3.

17. Olivotto I, Cecchi F, Yacoub MH. Myocardial bridging and sudden death in hypertrophic cardiomyopathy: Salome drops another veil. Eur Heart J 2009;30:1549-50.

18. Escaned J, Cortés J, Flores A, et al. Importance of diastolic fractional flow reserve and dobutamine challenge in physiologic assessment of myocardial bridging. J Am Coll Cardiol 2003;42:226-33.

19. Authors/Task Force members, Elliott PM, Anastasakis A, et al. 2014 ESC Guidelines on diagnosis and management of hypertrophic cardiomyopathy: the Task Force for the Diagnosis and Management of Hypertrophic Cardiomyopathy of the European Society of Cardiology (ESC). Eur Heart J 2014;35:2733-79.

20. Rapezzi C, Arbustini E, Caforio AL, et al. Diagnostic 
work-up in cardiomyopathies: bridging the gap between clinical phenotypes and final diagnosis. A position statement from the ESC Working Group on Myocardial and Pericardial Diseases. Eur Heart J 2013;34:1448-58.

21. Maron BJ, Yacoub M, Dearani JA. Controversies in cardiovascular medicine. Benefits of surgery in obstructive hypertrophic cardiomyopathy: bring septal myectomy back for European patients. Eur Heart J 2011;32:1055-8.

22. Kim LK, Swaminathan RV, Looser P, et al. Hospital Volume Outcomes After Septal Myectomy and Alcohol Septal Ablation for Treatment of Obstructive Hypertrophic Cardiomyopathy: US Nationwide Inpatient Database, 2003-2011. JAMA Cardiol 2016;1:324-32.

23. Ommen SR, Maron BJ, Olivotto I, et al. Long-term effects of surgical septal myectomy on survival in patients

Cite this article as: Yacoub MH, Afifi A, Saad H, Aguib H, ElGuindy A. Current state of the art and future of myectomy. Ann Cardiothorac Surg 2017;6(4):307-317. doi: 10.21037/ acs.2017.06.04 with obstructive hypertrophic cardiomyopathy. J Am Coll Cardiol 2005;46:470-6.

24. Nielles-Vallespin S, Khalique Z, Ferreira PF, et al. Assessment of Myocardial Microstructural Dynamics by In Vivo Diffusion Tensor Cardiac Magnetic Resonance. J Am Coll Cardiol 2017;69:661-76.

25. Torii R, El-Hamamsy I, Donya M, et al. Integrated morphologic and functional assessment of the aortic root after different tissue valve root replacement procedures. J Thorac Cardiovasc Surg 2012;143:1422-8.

26. Lloyd R, Grover S, Parnham SF, et al. Preclinical alterations in cardiac energetics amongst sarcomere mutation carriers in hypertrophic cardiomyopathy. J Cardiovasc Magn Reson 2015;17:O83. 